\title{
Development and application of the active surveillance of pathogens microarray to monitor bacterial gene flux Richard A Stabler ${ }^{1}$, Lisa F Dawson ${ }^{1}$, Petra CF Oyston ${ }^{2}$, Richard W Titball ${ }^{3}$, Jim Wade ${ }^{4}$, Jason Hinds ${ }^{5}$, Adam A Witney ${ }^{5}$ and Brendan W Wren*1
}

\begin{abstract}
Address: ${ }^{1}$ Department of Infectious and Tropical Diseases, Keppel Street, London School of Hygiene and Tropical Medicine, London, WC1E 7HT, UK, ${ }^{2}$ Biomedical Sciences, DSTL, Porton Down, Salisbury, SP4 0JQ, UK, ${ }^{3}$ School of Biosciences, The University of Exeter, The Queen's Drive, Exeter, Devon, EX5 5QJ, UK, ${ }^{4}$ King's College Hospital, Denmark Hill, London, SE5 9RS, UK and ${ }^{5}$ Bacterial microarray group, St. George's, University of London, Cranmer Terrace, London, SW17 ORE, UK

Email: Richard A Stabler - richard.stabler@lshtm.ac.uk; Lisa F Dawson - lisa.dawson@lshtm.ac.uk; Petra CF Oyston - pcoyston@dstl.gov.uk; Richard W Titball - r.w.titball@exeter.ac.uk; Jim Wade - jim.wade@kch.nhs.uk; Jason Hinds - j.hinds@sgul.ac.uk;

Adam A Witney - awitney@sgul.ac.uk; Brendan WWren* - brendan.wren@lshtm.ac.uk

* Corresponding author
\end{abstract}

Published: 9 October 2008

BMC Microbiology 2008, 8:177 doi:10.1 I86/I47I-2180-8-177

This article is available from: http://www.biomedcentral.com/I47I-2180/8/177

(C) 2008 Stabler et al; licensee BioMed Central Ltd.

This is an Open Access article distributed under the terms of the Creative Commons Attribution License (http://creativecommons.org/licenses/by/2.0), which permits unrestricted use, distribution, and reproduction in any medium, provided the original work is properly cited.
Received: 25 June 2008

Accepted: 9 October 2008

\begin{abstract}
Background: Human and animal health is constantly under threat by emerging pathogens that have recently acquired genetic determinants that enhance their survival, transmissibility and virulence. We describe the construction and development of an Active Surveillance of Pathogens (ASP) oligonucleotide microarray, designed to 'actively survey' the genome of a given bacterial pathogen for virulence-associated genes.
\end{abstract}

Results: The microarray consists of 4958 reporters from I5I bacterial species and include genes for the identification of individual bacterial species as well as mobile genetic elements (transposons, plasmid and phage), virulence genes and antibiotic resistance genes. The ASP microarray was validated with nineteen bacterial pathogens species, including Francisella tularensis, Clostridium difficile, Staphylococcus aureus, Enterococcus faecium and Stenotrophomonas maltophilia. The ASP microarray identified these bacteria, and provided information on potential antibiotic resistance (eg sufamethoxazole resistance and sulfonamide resistance) and virulence determinants including genes likely to be acquired by horizontal gene transfer (e.g. an alpha-haemolysin).

Conclusion: The ASP microarray has potential in the clinic as a diagnostic tool, as a research tool for both known and emerging pathogens, and as an early warning system for pathogenic bacteria that have been recently modified either naturally or deliberately.

\section{Background}

Despite advances in the treatment of infectious disease, pathogenic bacteria represent one of the most important threats to health worldwide. Many infectious disease agents have never been controlled or have re-emerged as global pathogens, while others pose a new threat.
Recently emerged pathogens of heightened virulence include Vibrio cholerae O139 strains [1,2], Escherichia coli $0157[3,4]$, Salmonella enteritidis phage type-4 [5] and the multidrug resistant phage type-DT104, all of which have emerged over the last decade as a global health problem in both human and animal disease [6]. There is now clear 
evidence that these pathogens have become more virulent by acquiring genome segments through lateral gene transfer that result in gain-of-function traits [7]. This is dramatically illustrated by the transfer of antimicrobial resistance determinant among pathogenic (and non-pathogenic) bacteria such as meticillin resistant Staphylococcus aureus (MRSA) and multidrug resistant enterococci. The identification of newly acquired potentially harmful genetic elements and antibiotic resistances could influence clinical practice and policy, resulting in more effective treatment. Additionally, this identification of new traits could prove vital in the identification of bioterrorism threats, where the deliberate release of highly virulent pathogens is a major concern, particularly if the genome of the infectious agent has been modified with virulence determinants for nefarious purposes.

In the last few years, research in microbial pathogenesis and molecular epidemiology has changed fundamentally, from a piecemeal approach of characterising individual determinants or point mutations to a global analysis of pathogen genomes, fuelled by the ability to determine the complete genome sequence of microorganisms. This enabled the development of high-throughput nucleic acid hybridisation technologies including macro- and microarrays involving amplified gene fragments and oligonucleotide arrays (e.g. Affymetrix GeneChips). Microarrays have the capacity to rapidly monitor the genome content of bacterial strains and identify horizontal gene transfer elements, using thousands of reporter elements in a given experiment. Horizontal gene transfer is a major evolutionary mechanism for bacteria $[8,9]$, methods that can demonstrate gene acquisition (and loss) are crucial in identifying emerging pathogens, and could act as an early warning of the emergence of a potentially more virulent strain. At present microarray technology is limited to mainly single species microarrays. Yet the continued expansion of available genome sequence data and our increased understanding of the genetic basis of microbial virulence, this has presented a unique opportunity to monitor the genome content of microorganisms and the emergence of more virulent pathogens. With this in mind we report the development and application of an Active Surveillance of Pathogens (ASP) microarray for monitoring gene flux in pathogens, antimicrobial resistance and virulence profile, along with potentially identifying gene acquisitions and new outbreak strains. The ASP array is unique in that it represents known virulence determinants, antibiotic resistance genes and pathogenicity traits from 151 bacteria species, covering a broad range of species and genera on a single microarray. This provides an unparalleled opportunity to study gene flux and identify novel traits. The ASP microarray was validated with 19 bacterial species, ranging from those who's fully curated genome sequences were available, to those that were unsequenced, with little or no background information; including a blind control of a sample from unknown origins. The ASP array identified both known and unknown samples, including; Francisella tularensis, Clostridium difficile, Staphylococcus aureus, Vancomycin resistant Enterococcus faecium (VRE) and Stenotrophomonas maltophilia and provided information on drug resistance and the presence of potential virulence determinants.

\section{Methods}

Selection of oligonucleotide gene reporters for microarray Prior to the design of the oligonucleotides, a database of potential bacterial coding sequences (CDS) was established using published completed genome sequences; thus ensuring consistency in gene location and identity. A Perl script was used to extract CDS sequences from the sequence reference database (RefSeq) http://www.ncbi.nl m.nih.gov/projects/RefSeq/, which consists of curated completed and published genomes. Reporters for the ASP microarray were designed from CDS categorised by TIGR as either 'cell adhesion', 'detoxification', 'toxin production \& resistance' or 'pathogenicity' http://cmr.tigr.org/tigrscripts/CMR/shared/RoleList.cgi or TIGR Annotation search http://cmr.tigr.org/tigr-scripts/CMR/shared/Make FrontPages. cgi?page=searches \&crumbs=searches. Alongside these, additional reporters were designed based on ribosomal protein subunit genes, these were selected for their species, genus and family specificity. $16 \mathrm{~S} / 23 \mathrm{~S}$ genes were not used due to their high degree of conservation.

The oligonucleotide reporters were designed using OligoArray2.1 software [10]. Irrespective of GC content of the target genome the following parameters were set; GC content $40-60 \%$, Tm $85-90^{\circ} \mathrm{C}$ and runs of five homologous bases were prohibited. If OligoArray2.1 failed to design oligonucleotides for the majority of CDS, relaxed parameters were applied as follows; maximum GC-content was increased to $68 \%$ and maximum Tm increased to $94^{\circ} \mathrm{C}$ for high GC-content organisms. For low GC-content organisms the minimum GC-content was lowered to $35 \%$ and the minimum $\mathrm{Tm}$ decreased to $83^{\circ} \mathrm{C}$. Oligonucleotides were checked by BLASTN [11] using default settings but with a seed word length of seven (due to short oligonucleotide sequence length) and an e-value cut-off of 0.0001, against all CDS from all available species within a genera. If any oligonucleotides, had a match with a bit score of greater than 72 (approximately $>45 / 50$ bp match) to CDS other than that on which it was designed, the oligonucleotides for these CDS were considered redundant and thus removed i.e. one oligonucleotide will produce signal from homologous CDS, this accounts for duplicated genes or gene families. Subsequently a self-self BLAST for all selected oligonucleotides was performed to check for redundancy within the oligonucleotides, again any two oligonucleotides which shared greater than $45 / 50$ 
base pair identity were deemed to be a duplication and one was removed from the potential oligonucleotide list. This iterative approach allows for expansion of only novel reporter elements with the addition of each newly published genome sequence. The current ASP microarray (v5.3) consists of 4958 oligonucleotide reporters (additional file 1) designed from 99 bacterial genera (151 species, 205 genomes, plus at least 100 mobile genetic elements (MGE) additional file 3).

\section{Construction of ASP microarrays}

All oligonucleotides were synthesised by MWG Biotech (Ebersberg, Germany). Oligonucleotides were suspended in $50 \% \mathrm{DMSO}$ at a final concentration of $50 \mu \mathrm{M}$. Microarrays were constructed as described previously [12] by robotic spotting of oligonucleotides in duplicate on UltraGaps amino-silane coated glass slides (Corning, USA). The microarrays were post-print processed according to the slide manufacturer's instructions.

\section{DNA extractions}

Where strains were provided, they were grown in the recommended media, at the recommended temperature. DNA isolations was performed using either the wizard DNA extraction kit (Promega) or the Puregene DNA isolations kit (Gentra Systems) in accordance with the manufacturer's protocols for extraction of either Gram positive or Gram negative bacteria as appropriate. For C. difficile, the Puregene Gram positive extraction method was utilised with the addition of $175 \mu \mathrm{g}$ lysozyme to aid cell lysis, followed by snap freeze using a dry ice and ethanol bath. Samples were then heated to $80^{\circ} \mathrm{C}$ for $15 \mathrm{~min}$, and the manufacturer's protocol was then resumed.

\section{Hybridisation conditions \& data acquisition}

Test samples were labelled and hybridised as described previously [13] using 3-6 $\mu$ g DNA with a formamide based hybridisation buffer solution $(30 \%$ formamide, $3.75 \times$ Denhart's solution, $3.75 \times \mathrm{SSC}, 0.75 \mathrm{mM} \mathrm{Na}_{4} \mathrm{P}_{2} \mathrm{O}_{7}$, $37.5 \mathrm{mM}$ Tris pH 7.4, $0.075 \%$ SDS, $0.056 \mathrm{mg} / \mathrm{ml}$ used tRNA, modified from [14]) in a final volume of $48 \mu \mathrm{l}$ at $50^{\circ} \mathrm{C}$ for $16-20$ hours. The microarrays were washed as described previously but with the initial wash at $50^{\circ} \mathrm{C}$ [13]. The microarrays were scanned using a 418 microarray Scanner (Affymetrix, USA) and intensity fluorescence data acquired using ImaGene 5.5 (BioDiscovery, USA). After scanning, a commercial SpotCheck kit (Invitrogen) consisting of $\mathrm{Cy} 3$ labelled random 9-mers was used to hybridize to the microarray, which binds to all the reporters on the microarray. The microarray was then washed and scanned as before but without preheating the initial wash. This step is essential due to the small number of reporters, which hybridise with specific samples and serves as a valuable control to confirm microarray reporter presence on the microarray.

\section{Data analysis}

Initially positive reporter identification was attempted using the proprietary algorithms of ImaGene and BlueFuse software however these lacked the specificity and defined cut-offs required. The method described by Cassone et al. [15] was used to identify positive reporters. Each reporter was present on the ASP microarray in duplicate, for each replicate the median of the background signal was deducted from the reporter spot median fluorescence using Excel to give hybridisation signal (figure $1,2,3)$. The mean ( $\mathrm{m}$ ) and standard deviation (sd) of replicate hybridisation signals was calculated. Additionally the background corrected mean (M) and standard deviation (SD) for the whole microarray was calculated (figure 1, 2, 3). The data analysis methodology utilised two main calculations to determine whether a particular reporter was present in any given strain. Firstly, for true positives the signal should be consistent between microarray replicates, therefore if the signal mean was greater than the standard deviation of the spot intensities for a particular reporter ( $\mathrm{m}>\mathrm{sd}$ ) this was given a pass, whereas $\mathrm{sd}>\mathrm{m}$ was given a fail. Secondly, most of the reporters on the microarray do not produce signal, therefore a true positive reporter must be significantly brighter than the background reporters, therefore if the reporter signal mean (m) was greater than the microarray mean M plus SD $(\mathrm{m}>\mathrm{M}+\mathrm{SD})$ this was given a pass and $\mathrm{m}<\mathrm{M}+\mathrm{SD}$ a fail (figure $1,2,3)$. Reporters were considered present if given a pass for both tests. The resultant gene lists were then entered into GeneSpring. Subsequently in GeneSpring the list of positive reporters was compared to a database of lists consisting of: BLAST predictions, results from previous hybridisations and lists of all the oligonucleotides from each species present in the design database. This identified similarities between gene lists and hybridisation results. For each reporter list, the hypergeometric probability (p-value) was calculated. This probability indicates the level of similarity between gene lists, whereby the p-value was the probability that two gene lists ( $\mathrm{n} \& \mathrm{~m}$ ) share a subset of genes (k) if randomly selected from all ASP microarray genes (u). A low p-value indicates that the lists were not similar by chance.

$$
\frac{1}{\left(\begin{array}{c}
u \\
m
\end{array}\right)} \sum_{i=k}^{n}\left(\begin{array}{c}
m \\
i
\end{array}\right)\left(\begin{array}{c}
u-m \\
n-i
\end{array}\right)
$$

The test gene list was then added to the database. The continual addition to this database enables comparison to be made between the database and future samples to predict gene content and some degree of identification, for both known and unknown samples. 


\section{F. tularensis SCHU S4}

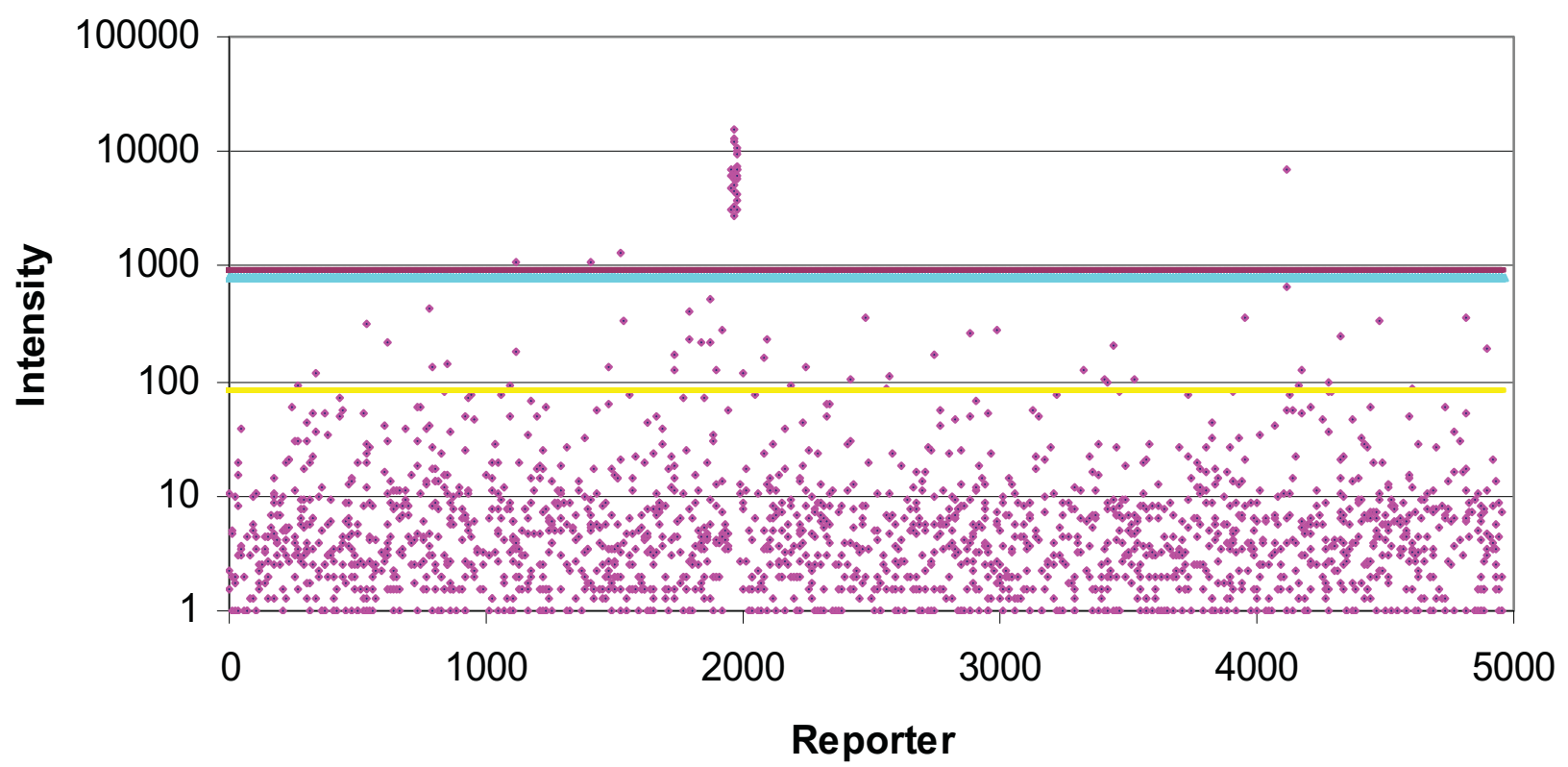

\section{Figure I}

Distribution of ASP reporter intensities and positive signal selection cut-offs for F. tularensis SCHU S4. Pink dots $=$ Individual ASP reporters (mean) signal, yellow line $=$ mean fluorescence of all ASP microarray reporters, light blue line $=$ one standard deviation (SD) of all ASP microarray reporters, mauve line = mean + SD, reporters with signal greater than mean+SD were selected as positive.

\section{Vectorette PCR}

Vectorette PCR [16] enables the amplification of specific DNA fragments where only one primer, in this case a 50 mer reporter, is known and was employed to validate the hybridisations. Genomic DNA was digested with either, AluI (Promega), EcoRV (Promega), PvuII (Promega) or RsaI (NEB) and heat-inactivated where appropriate, in accordance with the manufacturer's protocol. The vectorette bubble unit was prepared by annealing the oligonucleotides LDF1F and LDF1R (additional file 2). The vectorette unit was then ligated to the individual digested DNA, using T4 ligase (Promega) in accordance with the manufacturer's protocol. Nested 30-32 mer oligonucleotides were designed from within the 50 mer microarray reporter (additional file 2 ), along with nested primers complimentary to the vectorette bubble (additional file 2). Primary PCR reactions were performed using the vectorette internal primer LDF2, and the gene specific primer ( $5 \mu \mathrm{M}$ of each primer). The PCR reactions were performed using 2.5 units of Taq polymerase (Promega) in accordance with the manufacturer's protocol, 35 cycles of $92^{\circ} \mathrm{C}$ $30 \mathrm{sec}, 38^{\circ} \mathrm{C} 1 \mathrm{~min}, 72^{\circ} \mathrm{C} 1 \mathrm{~min}$ and a final cycle $72^{\circ} \mathrm{C} 10$ min. The secondary PCR was performed using the primary PCR reaction, with nested gene specific primer and nested vectorette primer. A total of 40 cycles of $92^{\circ} \mathrm{C} 30 \mathrm{sec}$, $55^{\circ} \mathrm{C} 1 \mathrm{~min}$ and $72^{\circ} \mathrm{C} 1 \mathrm{~min}$ with a final cycle $72^{\circ} \mathrm{C} 10$ min. The secondary PCR products were separated on an agarose gel and gel extracted using the QiaQuick gel extraction protocol (Qiagen) in accordance with the manufacturer's protocol. The purified PCR products were then cloned into pGEM (Promega) in accordance with the manufacturer's protocol and sequenced, using M13 forward and reverse primers (additional file 2). The resultant sequences were run through BLAST to identify the cloned genes.

\section{Results}

Choice of gene reporters for the ASP array

The ASP microarray was designed to function as a single Cy dye microarray, giving present/absent information about genes based on whether a reporter fluoresces or not. This is not a quantitative comparative assay, therefore two samples can be analysed independently on each microarray simultaneously by labelling one sample with $\mathrm{Cy} 3$ and 


\section{C. difficile 630}

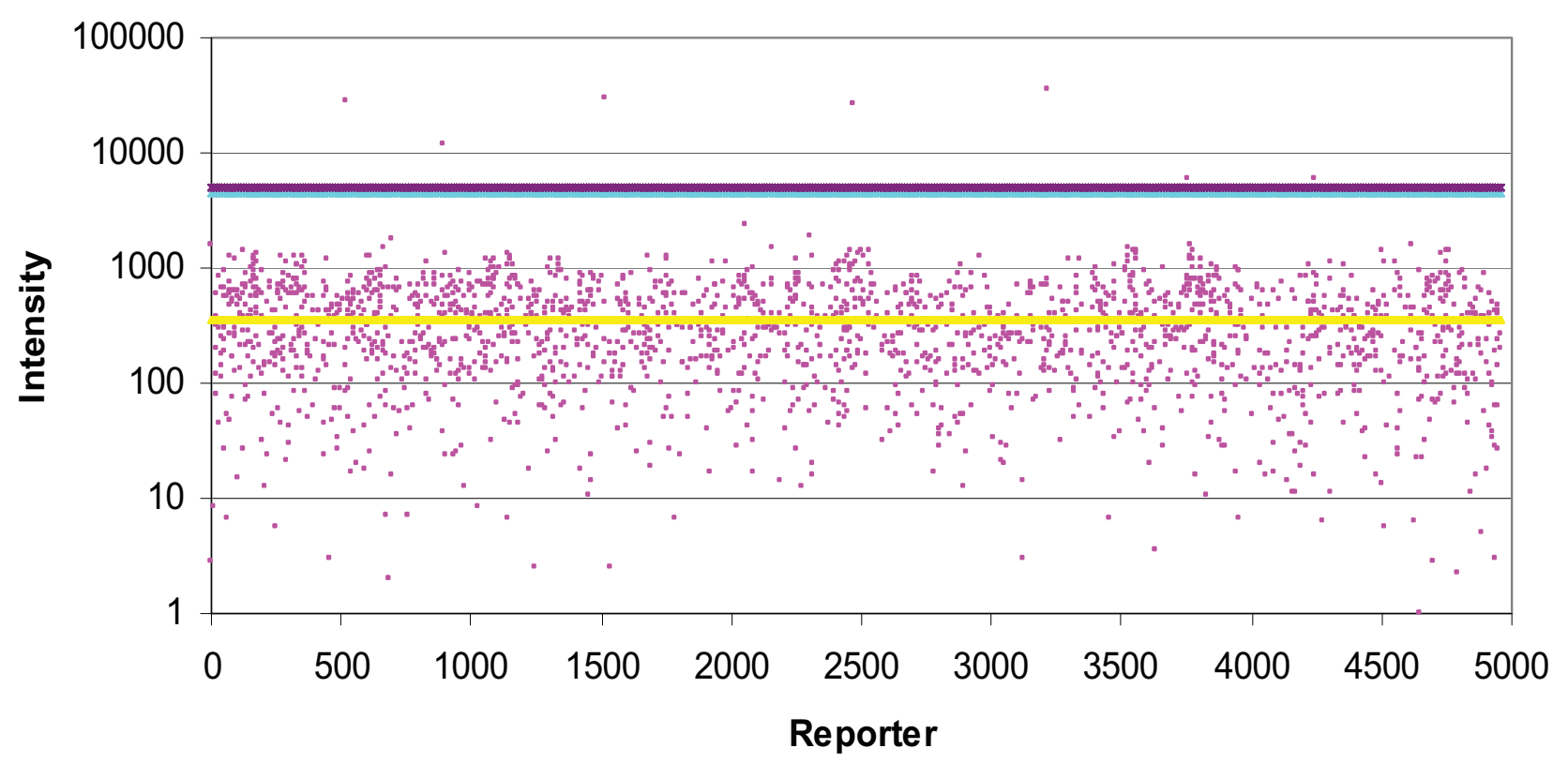

Figure 2

Distribution of ASP reporter intensities and positive signal selection cut-offs for $C$. difficile 630 . Pink dots $=$ Individual ASP reporters (mean) signal, yellow line = mean fluorescence of all ASP microarray reporters, light blue line $=$ one standard deviation (SD) of all ASP microarray reporters, mauve line = mean + SD, reporters with signal greater than mean + SD were selected as positive.

the other with Cy5. The concept microarray (ASPv1) was performed to compare oligonucleotides and PCR product microarrays using important pathogenic bacteria including Mycobacterium tuberculosis, S. aureus and Y. pestis (data not shown) and oligonucleotides were selected for use as reporters.

\section{Hybridisation of DNA from bacterial pathogens}

Validation was carried out using a selection of 19 bacteria (table 1) which fell into three "Rumsfeld" categories; 'known knowns' (KK) - consisting of fully sequenced and curated genome sequences, which enable BLAST predictions to be performed, 'known unknowns' (KU) - bacteria where partial or no sequence information is available, but for which limited information is available regarding species and drug resistances, and lastly 'unknown unknowns' (UU) - unsequenced bacteria with no prior information regarding species or drug resistance profile.

\section{"Known knowns"}

In order to validate the ASP microarray design and the bioinformatic analysis of data, a selection of sequenced isolates were used (table 1). The three examples presented in this study include F. tularensis SCHU S4, S. aureus COL and $C$. difficile 630. BLAST revealed that out of all the 4958 reporters on the microarray, F. tularensis SCHU S4 was predicted to hybridize to 27 reporters, S. aureus COL 107 (104 chromosomal and 3 plasmid) reporters, whereas $C$. difficile was predicted to hybridize to only two reporters. Hybridisation results were compared to BLAST predictions.

Hybridisation with F. tularensis SCHU S4 identified 29 positive reporters (figure 1), consisting of 27 predicted by BLAST. The two additional reporters were Cg34500228 and Cp39102563, however BLAST analysis of these reporters against the $F$. tularensis SHCU S4 genomes did not produce a match of greater than $22 / 26 \mathrm{bp}$, which was not predicted to be sufficient for hybridisation. 


\section{S. aureus COL}

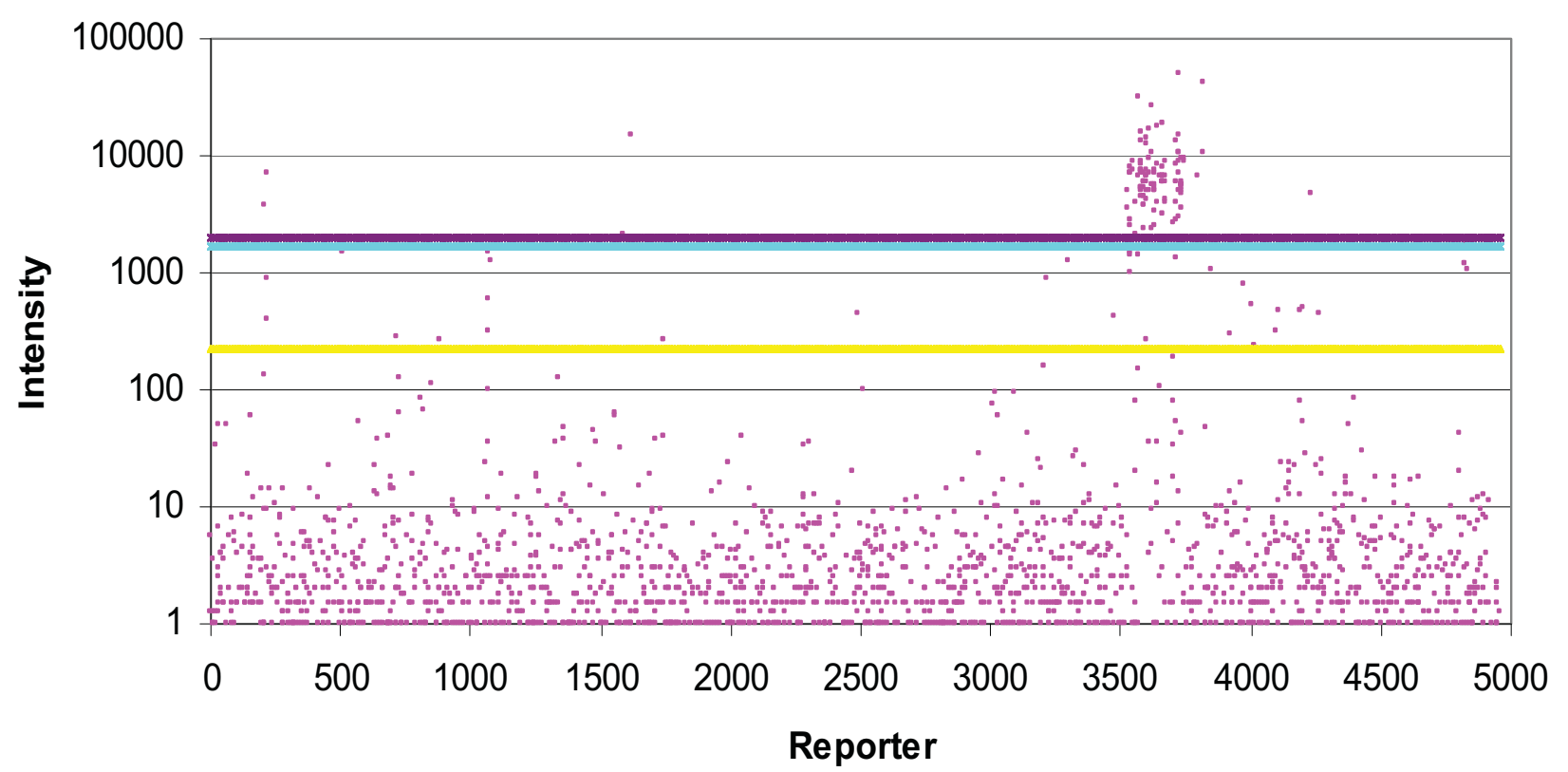

Figure 3

Distribution of ASP reporter intensities and positive signal selection cut-offs for $S$. aureus COL. Pink dots $=$ Individual ASP reporters (mean) signal, yellow line $=$ mean fluorescence of all ASP microarray reporters, light blue line $=$ one standard deviation (SD) of all ASP microarray reporters, mauve line $=$ mean $+S D$, reporters with signal greater than mean+SD were selected as positive.

Hybridisation with $S$. aureus COL DNA confirmed 101 of 107 BLAST predicted reporters in at least one of three hybridisations. Five predicted reporters produced weak signal just below the signal intensity cut-off and one produced negligible signal. Five additional reporters present by hybridisation were not predicted by BLAST. Four genes predicted to be present in $S$. aureus COL by hybridisation and/or BLAST were chosen for analysis by vectorette PCR to determine if the reporters were cross-hybridising to non-specific DNA. Haemolysin (Sa29510762) was used as a proof of principle control, it was positive in both BLAST prediction and hybridisation experiments. Exotoxin 3 (Sa29510468) was predicted by BLAST to be positive, yet the hybridisation failed for this reporter and finally two reporters positive by hybridisation but not predicted by BLAST; bleomycin resistance (Sa29520032) and chloramphenicol resistance (Cv50850700). The vectorette PCR amplified a band for the haemolysin, which was sequenced and proven to be the haemolysin gene (SACOL0762) (table 2). The exotoxin 3 was also confirmed as exotoxin 3 by vectorette PCR (table 2), suggesting the hybridisation conditions used for the arrays were not optimal for this specific reporter. Interestingly, the vectorette PCR for bleomycin and chloramphenicol resistance did not produce products with any of the four different $S$. aureus COL vectorette libraries (table 2), suggesting that the hybridisations on the microarray may have been due to miss-priming, or binding to non-specific DNA.

C. difficile strain 630 was used to test the microarray, there were no reporters specifically designed from $C$. difficile 630 , as only the pre-release genome sequence was available at the time of design and was therefore not included. C. difficile 630 CDS information was obtained from the pre-release genome using Artemis http:// www.sanger.ac.uk/Software/Artemis/ and BLAST analysis against the ASPv5.3 oligonucleotides was performed. Two reporters on the microarray (M50000021 and Sa41160923) had $100 \%$ identity to two genes within $C$. difficile 630, a duplicated erythromycin resistance transferase (Cd630-2007 \& -2010) and a tetracycline resistance gene (Cd630-0508). Hybridisations with C. difficile 630 genomic DNA revealed three positive reporters, the two mobile genetic elements as predicted, and the type III 
Table I: Bacterial species tested on the ASPv5.3 to date

\begin{tabular}{lllllll}
\hline $\begin{array}{l}\text { Bacterial } \\
\text { species }\end{array}$ & $\begin{array}{c}\text { Genome } \\
\text { available?' }\end{array}$ & $\begin{array}{c}\text { Rumsfeld } \\
\text { Category }\end{array}$ & $\begin{array}{c}\text { Ribosomal } \\
\text { predictions }\end{array}$
\end{tabular}

\begin{tabular}{|c|c|c|c|c|c|c|c|c|c|c|c|}
\hline $\begin{array}{l}\text { Acinetobacter baumannii } \\
(\gamma \text {-proteobacteria) }\end{array}$ & No & UU & no ribosomals & & 1 & 1 & & 1 & N/A $(0+12)^{4}$ & None $^{5}$ & - \\
\hline $\begin{array}{l}\text { Bacillus anthracis } \\
\text { (firmicutes) }\end{array}$ & Yes & KK & $\mathrm{n} / \mathrm{a}$ & I & 1 & & 3 & 3 & $4.43 \mathrm{e}-170(0+122)$ & B. cereus & $1.31 \mathrm{e}-133$ \\
\hline $\begin{array}{l}\text { Burkholderia mallei ( } \beta \text { - } \\
\text { proteobacteria) }\end{array}$ & Yes & KK & $\mathrm{n} / \mathrm{a}$ & & & & 3 & 3 & $1.55 \mathrm{e}-179(5+136)$ & B. pseudomallei & $1.34 \mathrm{e}-163$ \\
\hline $\begin{array}{l}\text { Burkholderia pseudomallei } \\
\text { ( } \beta \text {-proteobacteria) }\end{array}$ & Yes & KK & $\mathrm{n} / \mathrm{a}$ & & & & 3 & 3 & $6.61 \mathrm{e}-171(2+159)$ & B. mallei & $3.43 \mathrm{e}-110$ \\
\hline $\begin{array}{l}\text { Clostridium difficile } \\
\text { (firmicutes) }\end{array}$ & Yes & KK & $\mathrm{n} / \mathrm{a}$ & 1 & 1 & 9 & 12 & 4 & I.I le-4 $(0+3)$ & None & - \\
\hline $\begin{array}{l}\text { Clostridium sordellii } \\
\text { (firmicutes) }\end{array}$ & No & UU & no ribosomals & & & 1 & & 1 & $\mathrm{~N} / \mathrm{A}(0+2)$ & None & - \\
\hline $\begin{array}{l}\text { Clostridium tetani } \\
\text { (firmicutes) }\end{array}$ & No & UU & no ribosomals & & & 1 & & 1 & $\mathrm{~N} / \mathrm{A}(0+\mathrm{I})$ & None & - \\
\hline $\begin{array}{l}\text { Coxiella burnetii }(\gamma- \\
\text { proteobacteria) }\end{array}$ & Yes & $\mathrm{KK}$ & $\mathrm{n} / \mathrm{a}$ & & & & 2 & 3 & $3.32 \mathrm{e}-19(0+1 \mathrm{I})$ & None & - \\
\hline $\begin{array}{l}\text { Enterobacter sakazakii } \\
\text { (enterobacteriaeae) }\end{array}$ & No & UU & $\begin{array}{c}\gamma \text {-proteobacteria }(10 / 14 \\
\text { enterobacteriaceae })\end{array}$ & & & 14 & 11 & 14 & $N / A(10+13)$ & E. coli & $1.35 \mathrm{e}-11$ \\
\hline $\begin{array}{l}\text { Enterobacter cloacae } \\
\text { (enterobacteriaeae) }\end{array}$ & No & UU & $\begin{array}{c}\gamma \text {-proteobacteria }(10 / I I I \\
\text { enterobacteriaceae })\end{array}$ & & 1 & 1 & & 1 & $\mathrm{~N} / \mathrm{A}(3+19)$ & E. coli & $3.47 e-7$ \\
\hline $\begin{array}{l}\text { Entrococcus faecium } \\
\text { (firmicutes) }\end{array}$ & No & UU & Firmicutes & & 2 & 2 & 2 & & $N / A(I+I I)$ & S. aureus & $7.77 e-4$ \\
\hline
\end{tabular}




\begin{tabular}{|c|c|c|c|c|c|c|c|c|c|c|c|}
\hline $\begin{array}{l}\text { Escherichia coli }(\gamma- \\
\text { proteobacteria) }\end{array}$ & Yes & KU & $\mathrm{n} / \mathrm{a}$ & 1 & 1 & 1 & & 1 & $8.82 e-9(8+23)$ & S. flexneri & $1.35 \mathrm{e}-4$ \\
\hline $\begin{array}{l}\text { Francisella tularensis }(\gamma- \\
\text { proteobacteria) }\end{array}$ & Yes & KK & $\mathrm{n} / \mathrm{a}$ & & & & 3 & 3 & $7.33 e-65(3+24)$ & None & - \\
\hline $\begin{array}{l}\text { Klebsiella pneumoniae }(\gamma- \\
\text { proteobacteria) }\end{array}$ & No & UU & $\mathrm{n} / \mathrm{a}$ & & 1 & I & & 1 & $\mathrm{~N} / \mathrm{A}(0+8)$ & E. coli & $3.19 \mathrm{e}-4$ \\
\hline $\begin{array}{l}\text { Legionella pneumophilia } \\
\text { ( } \gamma \text {-proteobacteria) }\end{array}$ & Yes & KK & $\mathrm{n} / \mathrm{a}$ & & & & 3 & 3 & $7.08 \mathrm{e}-102(0+58)$ & None & - \\
\hline $\begin{array}{l}\text { Staphylococcus aureus } \\
\text { (firmicutes) }\end{array}$ & Yes & $\mathrm{KK} / \mathrm{KU}$ & $\mathrm{n} / \mathrm{a}$ & 2 & 1 & I & & 3 & $\begin{array}{c}8.18 \mathrm{e}-168(\mathrm{KK})(4+98) \\
5.87 \mathrm{e}-129(\mathrm{KU})(10+102)\end{array}$ & None & - \\
\hline $\begin{array}{l}\text { Stenotrophomonas } \\
\text { maltophilia }(\gamma- \\
\text { proteobacteria) }\end{array}$ & No & $\mathrm{KU}^{6}$ & proteobacteria $(\gamma$ - or $\beta$-) & & 1 & I & & I & $N / A^{6}(I+6)$ & None & - \\
\hline $\begin{array}{l}\text { Yersinia pestis }(\gamma- \\
\text { proteobacteria) }\end{array}$ & Yes & KK & $\mathrm{n} / \mathrm{a}$ & 3 & 2 & & & 2 & $1.35 e-219(11+188)$ & Y. pseudoTB & $5.32 \mathrm{e}-202$ \\
\hline $\begin{array}{l}\text { Yersinia } \\
\text { pseudotuberculosis }(\gamma- \\
\text { proteobacteria) }\end{array}$ & Yes & KK & $\mathrm{n} / \mathrm{a}$ & & & 3 & & 3 & $8.65 \mathrm{e}-199(10+129)$ & Y. pestis & $3.44 \mathrm{e}-188$ \\
\hline
\end{tabular}

$\mathrm{I}=$ Indicates availability of at least one sequenced strain at time of analysis. 2 = example p-value for correct prediction (on ASPv5.3), $3=$ example of best incorrect prediction and p-value (on ASPv5.3), $4=$ number of positive reporters from example hybridisation (ribosomals + 'virulence' genes), reporters from these genus/species underrepresented on ASPv5.3 due to lack of completed genome sequences however positive genes give information of bacteria (e.g. antibiotic resistance) and any positive ribosomal genes can be used to predict to the level of family/genus, $5=$ None indicates no significant match with any BLAST predictions within current database, $6=\mathrm{S}$. maltophilia sequence not available at time of analysis, $7=$ identification of UU's using ribosomal genes $(n / a=$ not appropriate if species can be elucidated), if present, based on the number of genera which the ribosome is present in at least I species (number of predicted genera including duplicates) 
Table 2: Summary of BLAST prediction, hybridisation data and vectorette PCR for putative S. aureus COL genes

\begin{tabular}{|c|c|c|c|c|c|}
\hline Gene Name/phenotype & Gene ID & BLAST/hyb & Vectorette product & Top Hit & Size \\
\hline Haemolysin & SACOL0762 & Yes/Yes & Yes & SACOL0762 & $126 / 126$ \\
\hline Exotoxin 3 & SACOL0468 & Yes/No & Yes & SACOL0468 & $362 / 362$ \\
\hline Bleomycin resistance & SAR0032 & No/Yes & No & $\mathrm{n} / \mathrm{a}$ & $\mathrm{n} / \mathrm{a}$ \\
\hline Chloramphenicol resistance & CV0700 & $\mathrm{No} / \mathrm{Yes}$ & No & $\mathrm{n} / \mathrm{a}$ & $\mathrm{n} / \mathrm{a}$ \\
\hline
\end{tabular}

secretion protein HrpT (Ps45781390) designed from Pseudomonas syringae DC3000. By BLAST, Ps45781390 had only a partial match to the C. difficile 630 genome. The presence of the two predicted genes in C. difficile 630 was confirmed using vectorette PCR (methods section) using the vectorette libraries derived from C. difficile 630.

\section{"Known unknowns"}

Eight clinical isolates were obtained from Kings College Hospital, London and had been identified to species level and tested for antimicrobial susceptibility using conventional methods. However, these pathogens had not been sequenced and therefore BLAST analysis could not be performed, but hybridisations results could be compared to BLAST predictions from other sequenced strains. These pathogens were used to test the ability of the ASP microarray to identify, distinguish and predict gene content. Two bacterial pathogens, S. aureus and E. faecium, are described here as examples.

The S. aureus strain tested on ASPv5.3 was a Panton-Valentine leukocidin (PVL) positive Community Acquired (CA)-MRSA with known antimicrobial resistances (ampicillin $^{\mathrm{R}}$, meticillin ${ }^{\mathrm{R}}$ and penicillin ${ }^{\mathrm{R}}$ ). Hybridisations identified 112 positive reporters on the ASP microarray which most closely resembles $S$. aureus strain MRSA252 (hypergeometric probability p-value of $5.87 \mathrm{e}^{-129}$ ) (table 1 ). The positive ASP reporters included four meticillin resistanceassociated genes and a $\beta$-lactamase precursor, which corroborates the known antimicrobial resistance phenotype. Additionally, putative resistances to bicyclomycin, bleo- mycin, fluoroquinolones, chloramphenicol, sufamethoxazole, and sulphonamide were identified. Although sensitive to antibacterial glycopeptides, this isolate carries two teicoplanin resistance-associated genes, BLAST analysis of these ASP reporters sequences identified matches with $100 \%$ identity in fourteen $S$. aureus complete genomes. Interestingly, both genes required for PVL and an additional leukocidin were also detected, which corroborates the known toxin phenotype. Additionally, a further 13 exotoxins were identified; an exfoliative toxin $\mathrm{A}$, an $\alpha$-haemolysin, $\gamma$-haemolysin (components A, B \& C), a putative haemolysin, a putative vacuolating cytotoxin and a $\beta$-haemolysin. Two $S$. aureus pathogenicity islands were partially identified, one from the bovine $S$. aureus RF22 (5/7 reporters) and the other from $S$. aureus COL (3/9 reporters). Of interest were 12 non-S. aureus reporters, six of which were designed from plasmids (table 3 ). This indicates that the ASP microarray has identified genes that are potentially novel to $S$. aureus, which may have been acquired by horizontal gene transfer (table 3 ).

The two vancomycin-resistant E. faecium (VRE) isolates, distinguishable by tetracycline ${ }^{\mathrm{R}}(\mathrm{KCH} 1)$ and linezoid ${ }^{\mathrm{R}}$ (KCH2), were both resistant to ampicillin, erythromycin, gentamicin, penicillin, rifampicin and trimethoprim. DNA from both isolates were tested in duplicate on the ASP microarray. ASPv5.3 did not contain any specific $E$. faecium reporters, however the microarray did contain 40 reporters from the related species E. faecalis (strain V583). As no E. faecium strains have been sequenced it was not possible to predict which, if any, E. faecalis V583 reporters

Table 3: Potential horizontally acquired genes predicted by ASP microarray in a clinical CA-MRSA isolate

\begin{tabular}{llll}
\hline Reporter & Annotation & Information source & Location \\
\hline Ba693320065 & Type IV secretion system protein VirB5 & Brucella abortus & Chromosome \\
Bq595505520 & Alpha-hemolysin & Bartonella quintana & Chromosome \\
Bq595505860 & Invasion associated locus B (lalB) protein family & Bartonella quintana & Chromosome \\
Cv50850766 & Probable multidrug resistance protein & Chromobacterium violaceum & Chromosome \\
M494500I5 & Ethidium bromide resistance protein QacEdeltal & Corynebacterium glutamicum & Plasmid \\
M49450016 & Sufamethoxazole resistance protein Sull & Corynebacterium glutamicum & Plasmid \\
M49730008 & Sulfonamide-resistant dihydropteroate synthase & Uncultured eubacterium & Plasmid \\
Nm31I62I46 & Neisseria-specific antigen protein, TspA & Neisseria meningitides & Chromosome \\
pFCMIAmp & Ampicillin resistance & Cloning vector (Tn7 based) & Plasmid \\
PRSBI0II5Su & Dihydropteroate synthetase type I confers sulfonamide resistance & Uncultured eubacterium & Plasmid \\
pRSBI0II6Qac & Quaternary ammonium resistance & Uncultured eubacterium & Plasmid
\end{tabular}


would hybridise. Hybridisations on the ASP microarray identified eight genes that were present in both strains of VRE, including five vancomycin resistance related genes, four of which matched the vancomycin ${ }^{\mathrm{R}}$ operon genes found on the $S$. aureus plasmid pLW043. The other four hybridisation positives were to an erythromycin resistance transferase and two genes from the related organism $E$. faecalis; an adhesion lipoprotein and a S14 ribosomal protein. The ribosomal reporter S14 was used for identification of the bacteria, however the discriminatory power of this particular ribosomal reporter was limited to the phylum firmicutes due to sequence homology from bacteria within two different orders (Bacillales and Lactobacillales). The reporter list for VRE KCH1 contained the tetracycline reporter, which was absent in the reporter list for the second test sample VRE KCH2; thus ASP data corresponds to the known sensitivities of these bacteria as indicated above. BLAST analysis of the tetracycline reporter produced a $100 \%$ match to a $E$. faecium tetracycline resistance gene (accession number AY081910). VRE KCH1 ASP hybridisation results also identified potential resistance conferred by streptothricin acetyltransferase and streptomycin aminoglycoside 6-adenyltransferase. However, susceptibility to these agents is not routinely determined. To confirm this in-vitro, susceptibility testing was performed using streptomycin on both VRE, each isolate exhibited an MIC of $>128 \mathrm{mg} / \mathrm{L}$.

The reporter lists for both of the VRE strains have been incorporated into the gene lists in GeneSpring, which importantly, this will expedite the identification of future VRE isolates.

\section{"Unknown unknowns" Stenotrophomonas maltophilia, a member of the $\gamma$-proteo- bacteria, is a pathogen found in immunocompromised patients and is naturally resistant to many broad-spec- trum antimicrobials, making it often difficult to treat. Until the very recent publication of the S. maltophilia K279a genome sequence in 2008 [17] little genetic infor- mation was available for this organism. A clinical isolate of S. maltophilia was obtained from the Kings College Hos- pital with no information on the antimicrobial suscepti-}

bility profile. The reporter list identified it as a $\beta$ - or $\gamma$ proteobacteria species by the ribosomal reporter (Bp63503218). Six further reporters were linked to antimicrobial resistance and detoxification (table 4), which may give an insight into the drug resistances of this isolate of $S$. maltophilia. For example reporter (Ps70052483) was annotated as conferring acriflavin resistance and $S$. maltophilia ULA-511 has been demonstrated to be acriflavin resistant [MIC $256 \mu \mathrm{g} / \mathrm{ml}$ ] [18]. The subsequent release of the S. maltophilia K279a genome, allowed a BLAST analysis to be performed, which confirmed a match of between $43 / 50$ bp to $47 / 50$ bp for five out of seven reporters (table 4). The remaining two reporters were specific to this clinical isolate.

The ASP microarray in this case was able to identify the bacterium to either a $\beta$ - or $\gamma$-proteobacteria class, and importantly provided some insight into the potential antibiotic resistance profile of this understudied organism. This positive reporter list was added into the hybridisations database to expedite the distinction of S. maltophilia from other bacteria.

\section{Classification of unknown bacteria using ribosomal reporters}

Ribosomal reporters were added to the ASP microarray to aid identification of test organisms, however $16 \mathrm{~S}$ and $23 \mathrm{~S}$ were not selected due to their high level of conservation among species. The discriminatory power of the ribosomal reporters was assessed in Table 1. For example, a test organism identified eleven ribosomal genes (table 5) on the ASP microarray, these ribosomal reporters were then used in a BLAST analysis, which identified 11 bacterial genera, all predicted by BLAST to contain at least one of the ribosomal reporters (table 5). All of these 11 bacterial genera fell within the $\gamma$-proteobacteria. Further analysis revealed that six of the genera are all members of the Enterobacteriaceae family, however, more importantly all eleven reporters are only present in the Yersiniae (table 5). Furthermore, one reporter (Yp58100797) appears to be specific to the Yersiniae (table 5), therefore using only the ribosomal reporters only, it suggests that the test organism was a member of the $\gamma$-proteobacteria and most likely of the genus Yersinia.

Table 4: Positive ASP microarray reporters from a clinical isolate of S. maltophilia

\begin{tabular}{llc}
\hline Reporter & Annotation & BLAST match with S. maltophilia K279a \\
\hline As65131772 & Bacterial type II secretion system protein E & $43 / 50$ \\
Bp63503218 & 30S ribosomal protein S12 & $45 / 50$ \\
Bt76501375 & Heavy metal efflux pump CzcA & $43 / 50$ \\
Pf41290909 & Superoxide dismutase, Mn & $47 / 50$ \\
Ps70052483 & Acriflavin resistance protein & $45 / 50$ \\
Se6905I442 & Secretion system apparatus SsaT & no hit \\
Tt3869II42 & ABC-type multidrug transport system, permease component & no hit
\end{tabular}

Five reporters predicted by ASP were also present in the S. maltophilia $\mathrm{K} 279$ a sequence strain but two genes were specific to the clinical isolate 
Table 5: Ribosomal reporters identified in an unknown isolate ( $Y$. pseudotuberculosis) and their predicted conservation within the prokaryotes

\begin{tabular}{|c|c|c|c|c|c|c|c|c|c|c|c|}
\hline Reporter & Enterobacter & Erwinia & Escherichia & Haemophilus & Pasteurella & Photorhabdus & Salmonella & Shewenella & Shigella & Yersinia & Vibrio \\
\hline Family & E & E & E & $P$ & $P$ & E & E & S & $E$ & E & V \\
\hline $\mathrm{Hi} 09070800$ & & & & $Y$ & $Y$ & & Y & & & $Y$ & \\
\hline$V c 25052592$ & Y & Y & Y & & & & $Y$ & & $Y$ & $Y$ & $Y$ \\
\hline Vc25060290 & & & & & & & & $Y$ & & $Y$ & $Y$ \\
\hline Yp58100052 & Y & Y & Y & & & & Y & & $Y$ & $Y$ & \\
\hline Yp581001।2 & & & & & & & & & & $Y$ & \\
\hline Yp58100200 & & & Y & & & & & & $Y$ & $Y$ & \\
\hline Yp58100206 & Y & Y & $Y$ & & & Y & Y & & $Y$ & $Y$ & \\
\hline Yp581002|4 & & $Y$ & & & & & & & & $Y$ & \\
\hline Yp581002I5 & Y & & & & & & Y & & & $Y$ & \\
\hline Yp58100233 & Y & Y & Y & & & Y & Y & & $Y$ & $Y$ & \\
\hline Yp58100797 & & & & & & & & & & Y & \\
\hline Total (44) & 5 & 5 & 5 & I & I & 2 & 6 & I & 5 & 11 & 2 \\
\hline
\end{tabular}

$\mathrm{E}=$ Enterobacteriaceae, $\mathrm{P}=$ Pasteurellaceae, $\mathrm{S}=$ Shewenallaaceae, $\mathrm{V}=$ Vibrionaceae, $\mathrm{Y}=$ reporter identified in at least one species from that genera by BLAST analysis.

\section{Discussion}

The ASP array has validated over 823 gene sequences as suitable reporters from 19 pathogens. The reporters include both chromosomally located and plasmid encoded antimicrobial resistance cassettes, pathogenicity islands, secretion systems, effectors, toxins, drug export and detoxification systems designed from 99 different genera, 151 species, 205 genomes (additional file 3). In addition, accompanying reporters were designed from ribosomal proteins to aid in genera differentiation. This provides the opportunity to test an unlimited number of bacteria species to determine the content of their potential virulence determinants. One limitation of DNA hybridisations is that the presence of a gene reporter does not demonstrate that the gene is functional. For example a pathogen may carry a drug resistance gene or part of a resistance cassette/operon but may be currently sensitive to that antibiotic, however the presence of a nonexpressed resistance gene does indicate that there is a higher potential for resistance to be developed under exposure to the right antibiotics, which could influence choice of treatment antibiotic.

The ASPv5.3 microarray was tested and validated on a range of bacterial pathogens, from those with available genome sequences (e.g. F. tularensis SCHU S4, S. aureus COL and C. difficile 630) to those that are clinically important, but have limited information regarding antimicrobial resistance profile or genetic information, such as $S$. maltophilia. The $S$. aureus COL validation of the ASP microarray demonstrated that accurate prediction of positive reporters on the ASP microarray was achievable and that this data, confirmed by vectorette PCR, could be used successfully to identify bacteria with a very high level of certainty. The majority of predicted reporters produced positive signals, including plasmid-based genes, with only a few absent. As many antibiotic resistance genes are plasmid mediated, it is important to ensure extraction of both chromosomal and plasmid DNA for testing. In the case of $S$. aureus COL 3 BLAST predicted genes were located on the $S$. aureus COL plasmid pT181, all of which were positive in a least one hybridisation, suggesting that the 'chromosomal' preparation also extracted plasmid DNA, however the limitation is that the cannot distinguish chromosomal versus plasmid encoded. As predicted there were a few differences between observed (hybridisations) and predicted (BLAST) results, which did not adversely effect the identification of the bacteria. These differences can be attributed to hybridisations or bioinformatics errors. For such a large array, including reporters for a wide range of bacteria it was predicted that some of the reporters would fail, either in design, synthesis, microarray production or under the hybridisation conditions for the oligonucleotides. There are a number of ways of addressing the issue, however all have their limitations, one could increase stringency by increasing the value of the minimum positive signal cut-off to result in fewer false positives, yet this would produce more false negatives. The reducibility of the replicate spots is a critical factor for being defined as positive, greater accuracy could be achieved with either more replicates or multiple reporters per gene. However, duplicate reporters per gene would increase the cost of oligonucleotides, yielding little additional data. Both methods would greatly increase (up to double) the size of the microarray and physical space on the glass slide is finite. Improvements in printing density and microarray fabrication can currently manufacture up to $1,000,000$ reporters directly onto a slide for a fixed cost, which would enable a greater number of reporters, and, therefore the physical size of the microarray, but practically this may result in lower signal as the labelled DNA is diluted and dispersed over a greater area. Sensitivity, sig- 
nal strength and hybridisation time could be further improved by dynamic hybridisation using microfluid devises, rather than the static incubation used in this study. The bioinformatic analytical methodologies will be assessed and improved with the next version of the ASP array, along with an increase in the number of reporters per gene and the number of genes covered from an everexpanding variety of microorganisms for which genome sequence data is becoming available.

Analysis of C. difficile 630 hybridisations demonstrated useful genetic information can be obtained even when there were no specific reporters for an organism on the ASP microarray. Two of the positive reporters were designed from drug resistance genes present on mobile elements Tn916 in Streptococcus agalactiae 2603 V/R and the plasmid pRUM in E. faecium U37. These genes have been demonstrated to confer resistance to tetracycline and erythromycin in C. difficile 630 [19], which demonstrates that the ASP microarray can be used to obtain potential antimicrobial resistance information when the genome sequence is unavailable. The third positive reporter (Ps45781390) only had limited similarity with the C. difficile 630 chromosome, and was most likely cross-hybridizing to either an integrase (weak match (25/50)) match with an integrase or to the C. difficile 630 plasmid p630 (weak match (27/50), 1 gap). This reporter was most likely cross-hybridising. To improve the data from the ASP microarray for this important nosocomial pathogen, $C$. difficile specific reporters will be added to the next iteration of ASP microarray design.

Analysis of clinical isolates such as VRE and CA-MRSA confirmed that the ASP microarray could provide useful information in the identification process as well as important genetic information, such as pathogenicity traits and antimicrobial resistance, even when no VRE specific reporters were on the ASP microarray. Two clinical isolates of VRE were successfully distinguished through the presence of tetracycline resistance in one strain. The tetracycline reporter matched $100 \%$ to a previously described E. faecium resistance gene although it was not stated whether the gene was located chromosomally or on a plasmid, however this reporter was also found in vancomycin resistance genes located on two E. faecalis plasmids (pAM $\alpha 1$ and pJH1). The information gained from the hybridisation patterns obtained with these two strains improved the potential for correct identification of VRE from future samples, where information on strain or antimicrobial resistance profile is unavailable.

Analysis of the clinical CA-MRSA isolates was successfully identified as $S$. aureus, VPL-positive and meticillin resistant. Additional information revealed putative antimicrobial resistances and toxins previously unknown for these strains. Furthermore eleven genes encoding important drug resistance determinants were identified in the clinical isolate, using reporters from other species. Interestingly, six of which are known to be encoded on mobile elements suggesting horizontal gene transfer.

The genetics of the emerging pathogen S. maltophilia have only recently begun to be deciphered. However, the ASP microarray was able to identify seven genes, one ribosomal and six virulence-associated genes in a clinical isolate of $S$. maltophilia. Five out of seven reporters were subsequently confirmed to have matches in the recently published S. maltophilia K279a genome sequence [17] including the ribosomal and acriflavin resistance genes.

Sample identification was also proved successful with a blind test was performed on $Y$. pseudotuberculosis strain 0:56 using only information from the ribosomal reporters. These reporters identified $Y$. pseudotuberculosis as a $\gamma$ proteobacteria and most likely Yersinia (table 3). Additionally, the combination of ribosomal and virulence genes as a whole correctly distinguished this as $Y$. pseudotuberculosis $(\mathrm{p}=8.63 \mathrm{e}-199)$ from $Y$. pestis $(\mathrm{p}=3.44 \mathrm{e}-188)$. The ability to differentiate between these close relatives, indicates that the ASP microarray will also be able to differentiate between pathogens and closely related nonpathogenic species.

Inclusion of further antibiotic resistance markers will increase the utility of the ASP microarray for predicting the antibiotic resistance profile of unknown pathogens. As the microarray analysis can be undertaken more rapidly than traditional antibiotic susceptibility assays, this may help in guiding clinicians in their choice of therapy. For some pathogens, prompt initiation of antibiotic therapy in essential. For example, CDC guidelines in the treatment of plague are that antibiotic therapy should be initiated within $24 \mathrm{~h}$ of the development of symptoms. Currently it is possible to hybridise and analyse the ASP microarray in under 24 hours however this requires prior pathogen isolation, growth and DNA extraction and with the emergence of multidrug resistant strains of plague [2022 ] there is an obvious need to improve the turn around time. Improvements to the ASP microarray, with development of faster microarray technologies and automation along with direct detection from clinical specimens, would make the ASP microarray invaluable in administering appropriate antibiotic prescription.

Microarray-based technology is ideal for multiple gene identification and, therefore suited for rapid pathogen identification particularly antibiotic resistant strains. To this end, Berthnet et al. have recently adapted the Affymetrix resequencing GeneChip technology [23] to identify bacterial and viral species from within a complex (clini- 
cal) mixture and also identify and confirm the presence of antibiotic resistances. The advantage of resequencing is that it can identify point mutations in specific genes, including those responsible for antibiotic resistance. However the ASP microarray technology is cheaper and can identify a more comprehensive range of detectable genes.

Finally the concept of the ASP microarray design was to monitor the flow of genetic material between bacteria, both as an epidemiological tool and as a rapid screen of emerging pathogens. To achieve this the primary step is to ensure that 'normal' bacteria can be identified, along with expected virulence factors. This proof of principle paper establishes that the ASP microarray can do both, and has also provided data that some of the tested isolates contain unexpected genetic material demonstrating gene flux.

\section{Conclusion}

This proof of principle paper has demonstrated that the ASP microarray can be used to identify a wide variety of bacteria. More importantly, this system can also identify virulence-associated genes that have potentially been acquired by horizontal gene transfer. This ranges from information that could be relevant clinically (drug resistances and toxins) to the identification of potential genes that may be important in the emergence of virulent pathogens. The ASP microarray has demonstrated great potential and utility for the rapid surveillance of the gene content of a given bacterium, which could have uses clinically, in aiding diagnosis and identifying appropriate therapeutic regimens. The use may extend beyond the clinic, in following transfer of genes and the emergence of pathogens by pathogenicity island acquisition, for example, as observed by the spread of the Edinburgh-Toronto strain of Burkholderia cepacia in cystic fibrosis patients [24]. Additionally the ASP microarray can identify novel genes in a strain, which can be investigated using the reporter sequence and vectorette method. The ASP microarray is an adaptable tool, which will be continually modified, optimised and expanded to cover more pathogens including viruses and further virulence determinants, enhancing its impact and making it more generally applicable for diagnostic and research purposes.

\section{Abbreviations}

ASP: Active surveillance of pathogens; CDS: Coding sequence; MGE: Mobile genetic element; MRSA: Meticillin resistant Staphylococcus aureus; PVL: Panton-Valentine leukocidin; VRE: Vancomycin resistant Enterococcus faecium.

\section{Competing interests}

The authors declare that they have no competing interests.

\section{Authors' contributions}

RS designed, constructed and tested the ASP microarray, designed and optimised bioinformatics, set up GeneSpring database and helped draft the manuscript, LD tested the ASP microarray, carried out all vectorette work and helped draft the manuscript, PO, RT \& JW supplied strains and genetic material and provided scientific input, $\mathrm{JH}$ assisted with microarray design, AW assisted with design and PERL scripting, BW conceived the microarray and helped draft the manuscript. All authors have read and approved the final manuscript.

\section{Additional material}

\author{
Additional file 1 \\ ASP oligonucleotide reporters \\ Click here for file \\ [http://www.biomedcentral.com/content/supplementary/1471- \\ 2180-8-177-S1.xls]
}

\section{Additional file 2}

List of oligonucleotides used in the vectorette library construction, vectorette PCR and sequencing

Click here for file

[http://www.biomedcentral.com/content/supplementary/1471-

2180-8-177-S2.doc]

\section{Additional file 3}

ASP oligonucleotide reporters were designed from 99 different bacterial genera including 151 different species and 205 separate fully sequenced genomes.

Click here for file

[http://www.biomedcentral.com/content/supplementary/1471-

2180-8-177-S3.doc]

\section{Acknowledgements}

This work was funded by the MRC, DSTL and the Home Office, UK.

\section{References}

I. Faruque SM, Asadulghani, Saha MN, Alim AR, Albert MJ, Islam KM, Mekalanos J]: Analysis of clinical and environmental strains of nontoxigenic Vibrio cholerae for susceptibility to CTXPhi: molecular basis for origination of new strains with epidemic potential. Infect Immun 1998, 66( I 2):5819-5825.

2. Dziejman M, Balon E, Boyd D, Fraser CM, Heidelberg JF, Mekalanos $\mathrm{JJ}$ : Comparative genomic analysis of Vibrio cholerae: genes that correlate with cholera endemic and pandemic disease. Proc Natl Acad Sci USA 2002, 99(3): I556-I56I.

3. Prentice MB, James KD, Parkhill J, Baker SG, Stevens K, Simmonds MN, Mungall KL, Churcher C, Oyston PC, Titball RW, et al.: Yersinia pestis pFra shows biovar-specific differences and recent common ancestry with a Salmonella enterica serovar Typhi plasmid. J Bacteriol 200I, I 83(8):2586-2594.

4. Reid SD, Herbelin CJ, Bumbaugh AC, Selander RK, Whittam TS: Parallel evolution of virulence in pathogenic Escherichia coli. Nature 2000, 406(6791):64-67.

5. Wight JP, Cornell J, Rhodes P, Colley S, Webster S, Ridley AM: Four outbreaks of Salmonella enteritidis phage type 4 food poisoning linked to a single baker. Commun Dis Rep CDR Rev 1996, 6(8):RII2-II5. 
6. Cloeckaert A, Schwarz S: Molecular characterization, spread and evolution of multidrug resistance in Salmonella enterica typhimurium DT 104. Vet Res 200I, 32(3-4):30I-310.

7. Ochman $\mathrm{H}$, Lawrence JG, Groisman EA: Lateral gene transfer and the nature of bacterial innovation. Nature 2000, 405(6784):299-304

8. Karlin S, Campbell AM, Mrazek J: Comparative DNA analysis across diverse genomes. Annu Rev Genet 1998, 32: 185-225.

9. Pallen MJ, Wren BW: Bacterial pathogenomics. Nature 2007, 449(7 I 64):835-842.

10. Rouillard JM, Zuker M, Gulari E: OligoArray 2.0: design of oligonucleotide probes for DNA microarrays using a thermodynamic approach. Nucleic Acids Res 2003, 3 I (I 2):3057-3062.

II. Altschul SF, Madden TL, Schaffer AA, Zhang J, Zhang Z, Miller W, Lipman D): Gapped BLAST and PSI-BLAST: a new generation of protein database search programs. Nucleic Acids Res 1997, 25(I 7):3389-3402.

12. Hinds J, Witney AA, Vass JK: Microarray design for bacterial genomes. In Methods in Microbiology: Functional Microbial Genomics Edited by: Wren BW, Dorrell N. London: Academic Press; 2002:67-82.

13. Hinchliffe SJ, Isherwood KE, Stabler RA, Prentice MB, Rakin A, Nichols RA, Oyston PC, Hinds J, Titball RW, Wren BW: Application of DNA microarrays to study the evolutionary genomics of Yersinia pestis and Yersinia pseudotuberculosis. Genome Res 2003, I 3(9):2018-2029.

14. Petalidis L, Bhattacharyya S, Morris GA, Collins VP, Freeman TC, Lyons PA: Global amplification of mRNA by template-switching PCR: linearity and application to microarray analysis. Nucleic Acids Res 2003, 3 I (22): l 42.

15. Cassone M, D'Andrea MM, lannelli F, Oggioni MR, Rossolini GM, Pozzi G: DNA microarray for detection of macrolide resistance genes. Antimicrob Agents Chemother 2006, 50(6):2038-204I

16. Arnold C, Hodgson IJ: Vectorette PCR: a novel approach to genomic walking. PCR Methods Appl I99I, I(I):39-42.

17. Crossman LC, Gould VC, Dow JM, Vernikos GS, Okazaki A, Sebaihia M, Saunders D, Arrowsmith C, Carver T, Peters N, et al:: The complete genome, comparative and functional analysis of Stenotrophomonas maltophilia reveals an organism heavily shielded by drug resistance determinants. Genome Biol 2008 , 9(4):R74.

18. Zhang L, Li XZ, Poole K: SmeDEF multidrug efflux pump contributes to intrinsic multidrug resistance in Stenotrophomonas maltophilia. Antimicrob Agents Chemother 200I, 45(12):3497-3503.

19. Sebaihia M, Wren BW, Mullany P, Fairweather NF, Minton N, Stabler $\mathrm{R}$, Thomson NR, Roberts AP, Cerdeno-Tarraga AM, Wang $\mathrm{H}$, et al. The multidrug-resistant human pathogen Clostridium difficile has a highly mobile, mosaic genome. Nat Genet 2006, 38(7):779-786.

20. Guiyoule A, Gerbaud G, Buchrieser C, Galimand M, Rahalison L Chanteau S, Courvalin P, Carniel E: Transferable plasmid-mediated resistance to streptomycin in a clinical isolate of Yersinia pestis. Emerg Infect Dis 200I, 7(I):43-48

21. Galimand M, Carniel E, Courvalin P: Resistance of Yersinia pestis to antimicrobial agents. Antimicrob Agents Chemother 2006 , 50(10):3233-3236.

22. Welch TJ, Fricke WF, McDermott PF, White DG, Rosso ML, Rasko DA, Mammel MK, Eppinger M, Rosovitz MJ, Wagner D, et al.: Multiple antimicrobial resistance in plague: an emerging public health risk. PLOS ONE 2007, 2(3):e309.

23. Berthet $N$, Dickinson P, Filliol I, Reinhardt AK, Batejat C, Vallaeys T, Kong KA, Davies C, Lee W, Zhang S, et al.: Massively parallel pathogen identification using high-density microarrays. 2008 , I:79-86.

24. Sun L, Jiang RZ, Steinbach S, Holmes A, Campanelli C, Forstner J, Sajjan U, Tan Y, Riley M, Goldstein R: The emergence of a highly transmissible lineage of cbl+ Pseudomonas (Burkholderia) cepacia causing CF centre epidemics in North America and Britain. Nat Med 1995, I(7):66 |-666.
Publish with Bio Med Central and every scientist can read your work free of charge

"BioMed Central will be the most significant development for disseminating the results of biomedical research in our lifetime. "

Sir Paul Nurse, Cancer Research UK

Your research papers will be:

- available free of charge to the entire biomedical community

- peer reviewed and published immediately upon acceptance

- cited in PubMed and archived on PubMed Central

- yours - you keep the copyright

Submit your manuscript here:

http://www.biomedcentral.com/info/publishing_adv.asp
BioMedcentral 